\title{
ON A THEOREM OF O. HAUPT CHARACTERIZING PERIODS OF ABELIAN DIFFERENTIALS
}

\author{
HENRIK H. MARTENS
}

\section{Introduction}

In view of the notorious difficulty of characterizing the possible period matrices of closed Riemann surfaces (Schottky's problem), it is interesting to note that a very simple characterization of possible period rows was given by Otto Haupt in 1920 . In his paper [2] Haupt showed that a vector with complex entries $\left(w_{1}, \cdots, w_{g}, z_{1}, \ldots, z_{g}\right)$ is the period row of some holomorphic differential with respect to a canonical homology basis on some closed Riemann surface of genus $g$ if, and only if, the following two conditions are satisfied:

(a)

$$
i \sum_{j}\left(w_{j} \bar{z}_{j}-\bar{w}_{j} z_{j}\right)>0 \text {, and }
$$

(b) no transformation corresponding to a change of canonical basis will bring the vector to the form $(w, 0, \ldots, 0, z, 0, \ldots, 0)$.

In the case of a normalized vector on the form $\left(1,0, \ldots, 0, z_{1}, \ldots, z_{g}\right)$ the proof of the sufficiency of Haupt's conditions is particularly transparent, and the object of the present paper is to show this. We also derive a homology characterization of branched coverings of a torus given by Gerstenhaber [1] in the context of a discussion of Haupt's paper, but apparently with another proof in mind. Finally, we mention a connection with the problem of moduli. We do not treat the necessity of the conditions. It is easy to see that (a) follows from the Riemann period relations. Condition (b) lies deeper and is a special case of the fact that a jacobian variety cannot split in its canonical polarization.

\section{The basic construction}

The sufficiency of Haupt's condition can be established by an explicit construction of a Riemann surface.

Let $z_{1}$ be an arbitrary complex number with positive imaginary part, and consider the parallelogram in the complex plane with vertices $0,1,1+z_{1}, z_{1}$. By the 
usual identification of sides, the parallelogram may be turned into a torus. As a quotient surface the torus inherits a complex structure and a holomorphic differential with periods $\left(1, z_{1}\right)$ from the plane. Let $z_{2}$ be a point in the interior of the parallelogram or of one of the sides joining the origin to 1 or $z_{1}$. It is then possible to find two interior points of the parallelogram whose difference is $z_{2}$. A surface of genus 2 can now be constructed by cutting parallel slits of equal lengths centered at two such points and gluing the banks together to form a handle. (Corresponding points $z$ and $z+z_{2}$ of the slits are glued proceeding clockwise around one slit and counterclockwise around the other.) A canonical homology basis for this surface is given by the cycles of the torus corresponding to the sides of the parallelogram, and a new pair consisting of a closed curve surrounding one of the slits in the parallelogram and the cycle formed after the gluing process by the line joining the two points. It is clear that the holomorphic differential of the torus defines a holomorphic differential on the new surface with periods $\left(1,0, z_{1}, z_{2}\right)$ with respect to the indicated homology basis. Note that the corresponding endpoints of the slits are identified in this procedure and that the holomorphic differential on the new surface will have zeros at these points.

The above construction can obviously be repeated for $z_{3}, z_{4}, \ldots, z_{g}$, as long as the conditions of lying in the interior of the parallelogram or of the sides is satisfied. The interior points used in the construction may be chosen to be distinct, and the slits may be chosen small enough to lie in the interior of the parallelogram and not collide with each other. In this way a surface of genus $g$ with a holomorphic differential with periods $\left(1,0, \ldots, 0, z_{1}, \ldots, z_{g}\right)$ is obtained.

\section{Technicalities of reduction}

Suppose now that $\left(1,0, \ldots, 0, z_{1}, \ldots, z_{g}\right)$ is an arbitrary vector satisfying Haupt's conditions. Inequality (a) reduces in this case to $i\left(\bar{z}_{1}-z_{1}\right)>0$, which means that $z_{1}$ has positive imaginary part.

If $\left(1,0, \ldots, 0, z_{1}, \ldots, \ldots, z_{g}\right)$ is the period row of a holomorphic differential with respect to a canonical basis $A_{1}, \ldots, A_{g}, B_{1}, \ldots, B_{g}$, then the vector $\left(1,0, \ldots, 0, z_{1}, \ldots, z_{k}+m+n z_{1}, \ldots, z_{g}\right)$ is the period row of the same differential with respect to the basis

where

$$
A_{1}^{\prime}, \ldots, A_{g}^{\prime}, B_{1}^{\prime}, \ldots, B_{g}^{\prime}
$$

$$
\begin{gathered}
B_{k}^{\prime}=B_{k}+m A_{1}+n B_{1}, \\
B_{1}^{\prime}=B_{1}+m A_{k}, \\
A_{1}^{\prime}=A_{1}-n A_{k},
\end{gathered}
$$

and otherwise $A_{j}^{\prime}=A_{j}, B_{j}^{\prime}=B_{j}$. It is easy to verify that this is a canonical basis. Conversely, if the new vector is the period row of some holomorphic differential, so is the 
original one. It follows that the question of realizing a vector $\left(1,0, \ldots, 0, z_{1}, \ldots, z_{g}\right)$ as a period row is reduced to the question of realizing the vector $\left(1,0, \ldots, 0, z_{1}, z_{2}^{\prime}, \ldots, z_{g}^{\prime}\right)$ where $z_{k}^{\prime}=z_{k}+m_{k}+n_{k} z_{1}$ may be assumed to lie in the interior of the parallelogram defined by 1 and $z_{1}$, or on the sides joining the origin to 1 or $z_{1}$. This is almost the assumption underlying the basic construction, except that we may have $z_{k}^{\prime}=0$ for all $k \geqq 2$. This possibility is excluded by Haupt's condition (b). Thus by renumbering the cycles, if necessary, we may assume $z_{2}^{\prime} \neq 0$. If $z_{k}^{\prime}=0$ for some $k>2$, we may use the basis change

$$
\begin{gathered}
B_{k}^{\prime}=B_{k}+B_{2}, \\
A_{2}^{\prime}=A_{2}-A_{k}
\end{gathered}
$$

and otherwise $A_{j}^{\prime}=A_{j}, B_{j}^{\prime}=B_{j}$ to make $z_{k}^{\prime}=z_{2}^{\prime} \neq 0$. In this manner a vector satisfying the assumptions of the basic construction is produced, and the proof is complete.

\section{Branched coverings of tori}

Let $M$ be a $2 \times 2 g$ matrix of integers. Gerstenhaber [1] asserted without proof that there is a branched covering of a torus by a surface of genus $g$ such that $M$ represents the induced homology map with respect to canonical bases if, and only if, (a') $M J^{t} M=d\left(\begin{array}{rr}0 & 1 \\ -1 & 0\end{array}\right), d>0$, where $J=\left(\begin{array}{rr}0 & E \\ -E & 0\end{array}\right)$ is the intersection matrix of a canonical basis, and

( $\left.\mathrm{b}^{\prime}\right)$ no change of canonical bases would bring $M$ to the form

$$
\left(\begin{array}{l}
k, 0, \ldots, 0, m, 0, \ldots, 0 \\
l, 0, \ldots, 0, n, 0, \ldots, 0
\end{array}\right) \text {. }
$$

This result is an immediate consequence of Haupt's theorem. For, if $M$ represents a covering, we introduce complex structures on the surfaces by giving the torus an arbitrary complex structure and pulling it back to the covering surface. A differential with periods $(1, z)$ on the torus will then pull back to a differential with periods $(1, z) M$ on the covering surface, and it is trivial to see that conditions $\left(a^{\prime}\right)$ and $\left(b^{\prime}\right)$ correspond to conditions (a) and (b) applied to $(1, z) M$.

Moreover, the result proved here for normalized period rows suffices for the argument since any matrix $M$ satisfying conditions $\left(\mathrm{a}^{\prime}\right)$ and $\left(\mathrm{b}^{\prime}\right)$ may be transformed to the form

$$
\left(\begin{array}{l}
l, 0, \ldots, 0,0, n, 0, \ldots, 0 \\
0,0, \ldots, 0, m, 0,0, \ldots, 0
\end{array}\right), \quad l m>0, \quad n \neq 0,
$$

by a change of canonical basis [3]. With this form for $M$, any vector $(1, z)$ with $z$ in the upper half plane yields a vector

$$
(1, z) M=(l, 0, \ldots, 0, m z, n, 0, \ldots, 0)
$$


which is on the postulated form after dividing out $l$. If $\Pi$ is a period matrix for a Riemann surface of genus $g$ whose first row is $1 / l(1, z) M$, then the Hurwitz relation

$$
(l, 0 \ldots, 0) \Pi=(1, z) M
$$

defines a map of its jacobian variety onto the torus. The restriction of this map to the embedded Riemann surface is the desired covering map. More simply, the map is given by integrating the differential with period row $(1, z) M$.

\section{A question of moduli}

We shall say that a closed Riemann surface of genus $g \geqq 2$ admits a Haupt representation if it can be obtained from a torus by identifying slits as in the basic construction. Does every closed Riemann surface admit a Haupt representation? The answer is not clear (to me), but an affirmative one is strongly suggested by the following consideration:

In the construction of a surface of genus 2 from a given torus, it is clearly immaterial where the slits are placed, the essential variables are the separation $z_{2}$ and probably the length and orientation of the slit. These 2 complex parameters can be specified yielding, with $z_{1}$, three free parameters to determine a surface of genus 2 .

Introducing further slits to produce surfaces of higher genera, we have one parameter $z_{k}$, one parameter for the slit, and a third parameter for the location of the slit with respect to, say, the first slit. Thus each new handle involves the specification of three complex parameters, and the magic number $3 g-3$ appears.

Acknowledgment. I am grateful to the Department of Mathematics of the University of Copenhagen for generously putting an office at my disposal during a short sojourn at Copenhagen when this paper was written.

\section{References}

[1] Gerstenhaber, M.: On a theorem of Haupt and Wirtinger concerning the periods of a differential of the first kind, and a related topological theorem. - Proc. Amer. Math. Soc. 4, 1953, 476-481.

[2] Haupt, O.: Ein Satz über die Abelschen Integrale 1. Gattung. - Math. Z. 6, 1920, 219-237.

[3] Krazer, A.: Lehrbuch der Thetafunktionen. - B. G. Teubner, Leipzig, 1903, Ch. XI.

Norges Tekniske Høgskole

Department of Mathematics

N-7034 Trondheim

Norway

Received 1 November 1983 